\title{
Visualizing Time-course and Efficacy of In-vivo Measurements of Uterine EMG Signals in Sheep
}

\author{
Gaj Vidmar, Branimir L. Leskošek, Drago Rudel \\ University of Ljubljana, Faculty of Medicine, Institute of Biomedical Informatics \\ Vrazov trg 2, SI-1000 Ljubljana, Slovenia \\ \{gaj.vidmar, brane.leskosek, drago.rudel\}@mf.uni-lj.si \\ http://www.mf.uni-lj.si/ibmi-english
}

\begin{abstract}
A method for constructing condensed yet concise visualization of a long-term bioinformatics research project is presented. The chart combines information on data quality and chronology of research activities. It is implemented as Microsoft ${ }^{\circledR}$ Excel spreadsheet with VBA automation. The project comprised 3100 hours of EMG measurements in 23 pregnant sheep, each monitored for up to 5 months during pregnancy and immediately after labor. EMG signals were recorded from the surface of the uterine wall by electrodes implanted at the horn and cervix. The signals were assessed for quality, stored in electronic EMG archive and queried from there for visualization. The spreadsheet shows subject data as time-sorted column headings and pregnancy time along rows, with cell color depicting measurement efficacy rating (signal unusable, partly usable, OK), and a symbol marking labor date. The chart is best read if rotated into landscape format. Project group members confirmed usefulness of the chart in assessing research progress and spotting measurement problems.
\end{abstract}

\section{Introduction}

In the information society, the need for highly condensed yet accurate presentation of large amounts of categorized information for decision making purposes is ever increasing. The most inventive and successful methods and tools are both grounded in computer technology progress and inspired by the challenges it brings, like Treemap $[1,2]$, Holographic Research Strategy [3], or sparklines [4]. Such visualization often incorporates the time dimension, which is crucial in project management [5] or in the financial markets (www.panopticon.com). It is also important in other areas where a clear picture of complex system dynamics can improve the course of action, e.g., when monitoring hohspital activity via case mix [6]. Scientific projects in the field of biomedical informatics that involve years of high-tech, financially demanding and time-consuming measurements are another prime example. Hence, we aimed at constructing such a display as a tool for helping a long-term research project on electromyographic (EMG) activity of the uterus in pregnant sheep follow the success of the measurements. 
The whole project involved 35 sheep; the measurements addressed in the paper involved 28 pregnancies in 23 normally gravid sheep. All were multiparas with one or two fetuses. At low gestation (usually 30-60 days), each sheep was instrumented with EMG measuring equipment [7]. A pair of platinum electrodes was surgically attached to the uterine wall surface at the pregnant uterine horn, and the other pair at the cervix. The instrumented sheep were located in a stall with normal living conditions, where they moved around freely, even during measurements. Namely, two weeks after electrode implantation and veterinary care, the sheep were equipped with external EMG pre-amplifier worn in a belt around the body. During measurement, the pre-amplifier was wired to portable measuring equipment outside the stall.

As a rule, EMG measurements were performed at least twice a day, in the morning and in the afternoon, before labor, during labor and one week postpartum. In total, over 3100 hours of EMG recordings were made. A typical EMG recording session lasted for one hour during day, and for ten hours over night. The number of measurements per day in a sheep ranged from one to eleven, with few sheep subject to five or more sessions in a single day. The period for which a sheep was included in the research ranged from 3 to 134 days (median 74 days).

Two channels of EMG activity were recorded, sampled at $20 \mathrm{~Hz}$ frequency. The digitalized EMG signals were stored in the electronic archive database. To study various research hypotheses, the EMG data were mathematically processed in time, frequency and time/frequency domain. Subsequently, aggregated signal parameters were statistically analyzed.

Based on signal processing over one-minute intervals, each measurement session was visually assessed and classified into one of three categories:

- 0 - Whole record had to be removed from further analysis;

- 1 - Parts of the record had to be rejected;

- 2 - The whole record is OK.

In total, 3488 sessions were considered. For the horn, $66.9 \%$ of the sessions were rated as OK, $3.6 \%$ as partly usable, and $29.4 \%$ as unusable. For the cervix, the success rate was better: $75.6 \%$ of ratings were $2,2.4 \%$ were 1 , and $21.9 \%$ were 0 . The main reason for an EMG measurement session to fail was damage made to the electrode wires externally by the sheep.

\section{Method}

The chart is produced as Microsoft ${ }^{\circledR}$ Excel spreadsheet cell-chart with VBA automation. It requires the data to be placed on one sheet (in adjacent columns: subject name, measurement number, date, horn measurement efficacy rating, and cervix measurement efficacy rating; in the final column, the first row of data for each subject contains labor date). The chart is produced as a separate worksheet with gridlines hidden. The required data can be directly queried from our electronic EMG archive. The macro requests the user to input one parameter, the number of cells that represent one day (from 2 to 168, the default is 12). For our data, the default value of 12 was also the most suitable value in terms of trade-off between totally undistorted depiction (which would be ensured by divisibleness of the number by each 
encountered number of measurement sessions per day) and higher data-ink ratio [4] of the chart (which decreases with the number of cells per day).

The first two rows of the resulting worksheet are column headings with subject data (sheep name, date of labor, channel locations); the number of columns for each sheep corresponds to the number of channels used, and a narrow blank column separates the sheep. Within each channel column, each day (from the first to the last day of sheep's measurement period) is represented by a box: if there was no measurement on a given day, the box has neither border nor fill, so it is not drawn on the final chart; otherwise, the bordered box is vertically divided by the number of measurements per day (which is easily achieved by applying borders to worksheet cells given a sensibly chosen number of cells representing one day). Each box, i.e., pixel in terms of the pixelization paradigm $[6,8]$, is colored according to the measurement efficacy rating ( 0 - grey, 1 - yellow, 2 - white). A symbol in the empty column marks sheep's labor, so that the vertical time scale in days is anchored.

The final form of the chart is produced with the Microsoft ${ }^{\circledR}$ Excel's page setup feature by specifying that the document printout should be 1 page long and 1 page wide. For our dataset (with 12 cells per day), this just worked, but for bigger datasets, two-page long (or wide, or larger) charts should be produced (our VBA macro is limited just by maximum worksheet size). The chart can be conveniently exported for publishing or further processing by printing it to a PostScript printer assigned to a file (or using one of the many similar solutions). Readability is better if the chart is viewed in landscape format, so that subjects are represented by vertically stacked rows with time running in the horizontal direction, and in further discussion of the chart we refer to that orientation.

\section{Results}

The chart is presented in Fig. 1. From the header, we can read that the earliest measurement took place on 30 June, 1995, with sheep B, and that the last sheep included in the study was Ajda (her first measurement took place on 6 January, 2005). Next to the header, the letters $\mathrm{H}$ and $\mathrm{C}$ are put for the first and the last sheep, denoting horn and cervix measurements, respectively. Since the same pair of row headers applies to each sheep, the letters are omitted in-between for clarity.

The labor-denoting red arrow-like symbols placed at the end of the green vertical lines show that in three sheep, no labor took place. For F, Kaja (first pregnancy) and Mimi (first pregnancy), pregnancy must have terminated at some point in spontaneous abortion without the research staff or the farmer keeping the stall noticing, hence the cells with starting date of measurement for these sheep are colored grey rather than green. In the majority of the other sheep, the increased frequency and shorter duration of measurement sessions around labor, which was part of the study design, is clearly visible. Another observation quickly offered by the chart is that no successful measurement was obtained in only three pregnancies. A general message conveyed by the chart is that during the first half of the project, the follow-up periods were increasing, while the most important overall observation is that the share of completely successful measurement sessions increased over the course of the project. 


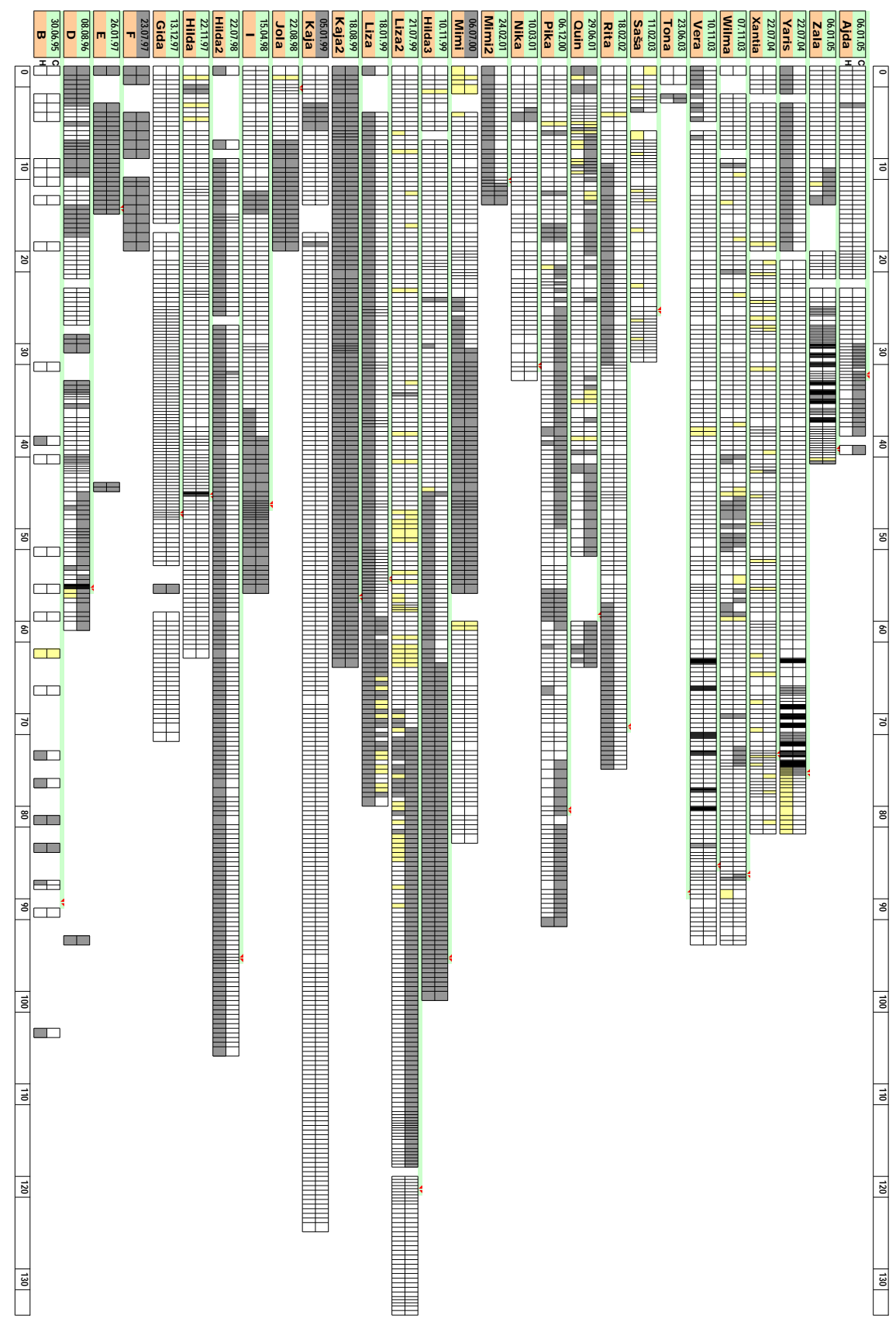

Fig. 1. Uterine EMG measurements in pregnant sheep over research project period 1995-2005. Sheep names run from B (first measured on 30 June, 1995) to Ajda (6 January, 2005); H= horn, $\mathrm{C}=$ cervix; red arrow shows date and time of labor (see Sect. 2 for details). 


\section{Discussion}

Our charting procedure is based on the Microsoft ${ }^{\circledR}$ Office suite on the Windows platform. As an alternative, a dedicated application for producing the chart could be developed, or it could be implemented in a cross-platform environment, e.g., as a $\mathrm{Java}^{\mathrm{TM}}$ applet, or in $\mathrm{R}$ [9]. Our spreadsheet-based implementation has natural limitations arising mainly from the issues of printer page size and resolution, but despite its relative simplicity it produces complex charts conveying large amount of information with minimum manual intervention from the user in just a few seconds on a standard personal computer.

The research-project group members confirmed the usefulness of the chart for displaying the quality of EMG recordings and thus presenting the success of their measurement efforts in general. The chart enabled them to spot in which sheep and in what time periods measurement problems occurred. Of course, such benefits cannot be quantified, but the problem spotting markedly aided in planning further sheep instrumentation and improving measurement set-up, and it is evident that the percentage of successful EMG measurement sessions subsequently increased. While the proposed visualization is primarily a tool for aiding research rather than a result of research, the chart for the present state of the whole EMG archive provides a summary of overall project progress that is useful and interesting in its own right.

In future research of uterine EMG, which is aimed at benefiting pregnant women in the long run, the chart's design principle could be incorporated into a graphic account of status of a single patient [10] or a group of patients. The presented visualization method can be applied to many fields of medical informatics, and in that sense, as well as in its design principles, it is similar to the generalized Caseview [8]. Namely, like the Caseview approach, it defines a reference frame based on three criteria: one nominal variable that can be meaningfully ordered (subject, sorted by date of entry into the study), one discrete or discretized dimension (time, which is treated as a ratio scale rather than just an ordinal one that is required by Caseview), and one binary attribute (used for splitting the frame in Caseview, but in our case it is measurement location and it defines row pairs). A further similarity with Caseview is ordinal color scale applied to the cells, while instead of numbers in cells used in Caseview, additional information is depicted with labor markers.

In conclusion, we would like to stress that the presented method should be used together with other visualization methods and amended whenever needed. The nature of contemporary data visualization is dynamic - in the words of one of its pioneers, Jacques Bertin [11]: "A graphic is no longer 'drawn' once and for all: it is constructed and reconstructed (manipulated) ... A graphic is never an end in itself: it is a moment in the process of decision making".

\section{References}

1. Shneiderman, B.: Tree visualization with tree-maps: 2-d space-filling approach. ACM Transactions on Graphics (TOG), 11(1) 92-99, 1992. 
2. Bederson, B.B., Shneiderman, B., Wattenberg, M.: Ordered and quantum Treemaps: making effective use of 2D space to display hierarchies. ACM Transactions on Graphics (TOG), 21(4) 833-854, 2002.

3. Végvári, A., Tompos, A., Göbölös, S., Margitfalvi, J.: Holographic research strategy for catalyst library design. Description of a new powerful optimisation method. Catalysis Today, 81(3) 517-527, 2003.

4. Tufte, E.: Beautiful evidence. Graphics Press, Chesire, CT (2005, in press).

5. Plaisant, C., Chintalapani, G ., Lukehart, C., Schiro, D., Ryan, J.: Using visualization tools to gain insight into your data. In: SPE Annual Technical Conference and Exhibition, 5-8 October, Denver, Colorado. Richardson, TX, SPE (2003) 1-9.

6. Lévy, P.P.: The case view, a generic method of visualization of the case mix. International Journal of Medical Informatics, 73(9-10) 713-718, 2004.

7. Leskošek, B., Pajntar, M., Rudel, D.: Time/frequency analysis of the uterine EMG in pregnancy and parturition in sheep. In: Magjarević, R. (ed.): Biomedical measurement and instrumentation - BMI'98. Proceedings of the 8th international IMEKO TC-13 conference on measurement in clinical medicine and 12th international symposium on biomedical engineering, Vol. 3. KoREMA, Zagreb (1998) 106-109.

8. Lévy, P.P., Duché, L., Darago, L., Dorléans, Y., , Toubiana, L., Vibert, J.-F., Flahault, A.: ICPCview: visualizing the International Classification of Primary Care. In: Engelbrecht, R., et al. (eds.): Connecting Medical Informatics and Bio-Informatics. Proceedings of MIE2005. IOS Press, Amsterdam (2005) 623-628.

9. $\mathrm{R}$ Development Core Team: $R$ : A language and environment for statistical computing. $\mathrm{R}$ Foundation for Statistical Computing, Vienna, Austria (2005). ISBN 3-900051-07-0, URL http://www.R-project.org

10.Powsner, S.M., Tufte, E.R.:Graphical summary of patient status. The Lancet, 344(8919) 386-389, 1994.

11.Bertin, J.: Graphics and graphic information-processing. de Gruyter, New York (1981). 